Research Article

\title{
Convergence of City Relational Network, Production Sector Structure, and Regional Development
}

\author{
Zunguo Hu $(\mathbb{D}$ and Jujing Zhou \\ School of Economics \& Management, Changsha University of Science \& Technology, Changsha 410114, China \\ Correspondence should be addressed to Zunguo Hu; 008003@csust.edu.cn
}

Received 31 May 2021; Revised 19 July 2021; Accepted 27 July 2021; Published 5 August 2021

Academic Editor: Guangdong Wu

Copyright (c) 2021 Zunguo Hu and Jujing Zhou. This is an open access article distributed under the Creative Commons Attribution License, which permits unrestricted use, distribution, and reproduction in any medium, provided the original work is properly cited.

\begin{abstract}
There is a strong correlation between government intervention and urban production structure in China. Particularly, the outputs of the cities partly come from the economic rent of city relational network (CRN), which is a unique regional policy and administrative hierarchy. In order to analyze the gravity flows of CRN under the nonmarket mechanism, we attempt to build a new gravity model that adopts the production sector. The new gravity produces relational data with direction, which makes it possible to use social network analysis (SNA) and overcome the endogeneity of the linear model. The empirical results show that (1) modified new gravity model can effectively capture the distribution of CRN gravity flows and the convergence of regional development in China, (2) the CRN, which especially stems from the government financial intervention, increases the share of nontradable sectors in cities, and (3) adjustment of the production sector leads to the difference of CRN gravity flows, so asymmetric flows distribution leads to the heterogeneity of regional economic performance. Cities with higher share of nontradables have relatively slower productivity growth in long-term.
\end{abstract}

\section{Introduction}

City relational network (CRN) is the network formed by the flows of people, logistics, and capital between regions. The economic rent of CRN is an important nonmarket source of outputs of Chinese cities, which is very different from urban governance in the market-oriented countries. For instance, despite the withering of tradable production sector, many undeveloped cities receive supports from regional coordination policies (e.g., transfer payment, industrial support, and preferential policy on infrastructure construction) and the income earned by migrant workers. In addition, the relocation of administrative officials and targeted support will also change the production sector. As a result, urbanization picks up speed in underdeveloped regions, which is featured by the expansion of the nontradable production sector. In other words, the relative structure of the production sector is altered by the economic rent of CRN. If the economic rent of CRN leads to a larger proportion of nontradable sectors, its long-term productivity will be slower than that of other cities with a larger proportion of tradable sectors. As a result, the regional development gap will be widened.

The two phenomena work together to reshape the regional economic trend. The correlations between Chinese cities have moved beyond the research scope of iceberg transport cost or nearest neighbor distance in new economic geography or spatial econometrics. The cities form a complex network involving interactions between people, logistics, and capital flow. Against this backdrop, this paper attempts to build a new gravity flow model for CRN, and reexamine the correlations of China's CRN through social network analysis (SNA). The research focuses on how the asymmetric changes of tradable and nontradable production sectors in different regions influence the convergence of regional development.

The asymmetric changes in the productivity of tradable and nontradable sectors in different regions have slowed down the convergence of regional development and even widened the development gaps. In developed regions, a large 
share of the production sector is tradable; the productivity of tradable production sector increases faster than the nontradable production sector $[1,2]$. Therefore, the tradable production sector attracts labor from other local sectors, and also draws high-skilled people from underdeveloped regions, causing a gradual decline of the tradable sector in underdeveloped regions. In addition, whether migrant workers make a living in other regions or return to their hometown for work, their surplus income leads to the phenomenon of Engel's consumption cycle, which stimulates the demand for nontradable products in their hometown, such as retail goods, real estate, and other services. In underdeveloped regions, the employment ratio of the nontradable production sector is further magnified by the local government's push for urbanization. The withering tradable sector and expanding nontradable sector in underdeveloped regions will tilt the balance of regional development $[3,4]$ : the relatively slow growth of the productivity of the nontradable sector adds to the difficulty for underdeveloped regions to catch up with developed regions. To overcome the imbalance of regional development, China has rolled out a series of top-down policies to balance the development across regions. The earliest policy is the Great Western Development Strategy, followed by the Northeast Area Revitalization Plan, and the Rise of Central China Plan. The ongoing urbanization process is also driven by many incentive policies [5]. The scale of a city is closely related to the local government. For example, many underdeveloped regions have issued various urbanization policies to attract population inflow. As a result, the employment ratio of the nontradable sector in these regions is poised to rise. By contrast, megacities like Beijing take lots of measures (e.g., tightening land supply) to limit the city scale. To sum up, the interregional trade of tradable products and the economic rent of CRN have both restructured regional production sector, and thus affected the convergence of regional development.

Under the combined effects of interregional trade and the economic rent of CRN, the relations between cities in China gradually form a complex network with multiple directions. However, there is hardly any report that discusses regional development based on the features of CRN, from the perspective of production sector restructuring. The few studies on regional economic convergence and spatial correlations mainly focus on the flow of factors [6], government roles [5], agglomeration economy, and even the reversal of enterprise agglomeration by the Internet [7]. Of course, some scholars have explored the close correlations between the employment and product price of tradable/ nontradable sector and regional productivity. The prices of nontradable products usually depend on the local market. In some parts of the nontradable sector, however, the rise of productivity will suppress the product prices. The price reduction mechanism clearly differs from the price drop of nontradable products induced by the economies of scale $[8,9]$. Trade liberalization can transmit product prices from the tradable sector to the nontradable sector $[10,11]$. He and Zhou [12] confirmed that international trade liberalization will squeeze out a portion of the formal employment in tradable and nontradable sectors, and promote the nonformal employment in the nontradable sector more effectively than that in the tradable sector. Similar studies have made great progress in the field of interregional trade. For instance, Shao et al. [13] empirically tested the close association between the regional linkage of economic development cycles and the trade of domestic value chain in China, and claimed that the division of labor in the value chain network is an important reason for the differences between the eastern region and the central/western region in internal and external spatial features. Using indices like the proportion and added value of service industry, Tang et al. [14] discovered the growing regional imbalance of the service industry in China: the eastern region is increasingly powerful, while the western region falls further back. In fact, the growing imbalance mentioned by Tang et al. [14] mainly refers to the widening gap of tradable service industry between regions.

Considering the current features of urbanization and the economic rent of CRN, it is difficult to fully explain the regional economic and social phenomena of China solely from traditional theoretical angles, such as free flow of factors. This paper attempts to build a statistical model based on the gravity flows in CRN, and uses the model to depict the structural features and convergence trend of the regional production sector, trying to make the analysis results more objective and accurate. Currently, the trade of tradable products between regions is usually measured by trade quality and trade intensity. The data on these attributes only reflect a side of the urban production sector. To fully analyze the complex interregional network of people flow, logistics, and capital flow, a good option is to replace the attribute data with relational data [15]. However, the replacement brings another problem: featured by relevance and directivity, the relational data go against the independence assumption in the measurement theory. Fortunately, this problem can be solved by the statistical methods related to social network analysis. Taylor et al. [16] and Fagiolo [17] shed new light to the academia through the topology inference of international trade and financial relational network; their estimation of parameter values and inference of relational network parameters (e.g., degree centrality and betweenness centrality) have won wide recognition. In recent years, great advancement has been made concerning the unobserved individual heterogeneity and network flow modeling for social and economic networks.

This work makes two marginal contributions. On the one hand, while the traditional gravity model centers on population size or per-capita gross domestic product (GDP), this paper modifies the gravity parameters, and introduces new variables into the benchmark gravity model (e.g., the ratio between tradable and nontradable sectors), creating directed gravity flows in the urban relational network. Our novel statistical model facilitates further research into the economic rent of CRN, breaks through the limitations of the proximity assumption in spatial econometric theory, and makes up for the lack of direction attributes in traditional data. Moreover, fitting tests were carried out by maximum likelihood estimation (MLE) and the iterative weighted least 
squares (IWLS) method derived from the Newton-Raphson method. The results show that our CRN gravity flow model and its method can effectively capture city structural changes and regional convergence trend of China. Previously, Li et al. [18] deduced the provincial spatial spillover effect through social network analysis combined with the Granger causality test of vector autoregressive (VAR) model. This combined approach is similar to the network graph statistics of Wasserman and Faust [19], and Scott [20]. However, the approach only vaguely selects the lag orders and processes the dynamic changes of spatial links, failing to explain the economic implication that affects sector adjustment and regional development. In this paper, the start and end point flows are quantified for each link in the $\mathrm{CRN}$, and the gravity model is corrected. These practices are inspired by the modeling ideas of Fagiolo [17], and Kolaczy and Csárdi [21] on national trade networks, as well as the use of gravity model by Liu et al. [22] to disclose the spatial clustering of energy consumption in China.

On the other hand, the empirical results show that the gravity flows to a city from other cities directly rely on the employment structure of that city (employment ratio of tradable sector to nontradable sector), and tend to increase significantly over time. This means that compared with underdeveloped cities, developed cities with a high share of tradable sector witness relatively fast growth in CRN gravity inflows. For simplicity, the tradable sector and nontradable sector are collectively referred to as the two sectors. Moreover, the per-capita GDP ratio between two cities increases with their employment structure ratio, that is, the two-sector employment ratio of a city divided by that of the other city. Therefore, the economic rent of CRN can increase the CRN gravity flows and the scale of nontradable sector in cities, pushing up the portion of that sector in the employment structure. Nevertheless, the cities with a high presence of nontradable sector are slower in the rise of overall productivity than those with a high presence of tradable sector, because the products of nontradable sector cannot be traded. The relatively slow growth of productivity in such cities would slow down the convergence of regional development. In addition, the growing productivity of the nontradable sector will lower the prices of some nontradable products, making the product between productivity and price in the nontradable sector unstable. As a result, it is not a feasible way for underdeveloped regions to expand the employment in cities only by improving the productivity of the nontradable sector.

The remainder of this paper is organized as follows: Section 2 models the adjustment of the urban production sector, and puts forward theoretical propositions; Section 3 presents ridge regression; Section 4 establishes a model of CRN gravity flows; and Section 5 summarizes the main findings.

\section{Benchmark Theories of Urban Production Structure}

Our theoretical model was constructed in reference to the ideas of Gollin et al. [3] concerning the equilibrium between such three sectors as the agricultural sector, the tradable production sector, and the nontradable production sector. The core of their research is to address the Dutch disease of resource-based country in the urbanization. However, our two sector model considers the structural adjustment and regional growth of only two urban production sectors, namely, tradable sector and nontradable sector. More importantly, the economic rent of CRN was introduced to the model, in the light of the unique urbanization background and regional development in China. The economic rent of $\mathrm{CRN}$ is manifested by the following phenomena: the cities on high administrative levels or large economic scales tend to concentrate lots of public resources (such as better health care and education). These cities earn much more through the attraction of people flow, logistics, and capital flow than the competitive market. Meanwhile, the economic rent of underdeveloped cities comes from the regional policy support by the central government, such as fiscal transfer, land supply, and preferential terms on infrastructure construction. Apparently, the economic rent of CRN has a close correlation with the variation in CRN gravity flows, and exerts an influence on the production sector adjustment and employment structure of cities. The urban production sector provides two types of outputs:

(1) Nontradable products

Nontradeable products refer to private services (local retail, transportation, education, and health) and other industrial products that cannot be traded in the national market. The price $P_{u}$ of such products depends on the endogeneities of the local market. Due to the nontradability, the growth rate of productivity of nontradable sectors is lower than that of tradable sectors.

(2) Tradable products

Tradable products refer to industrial products and tradable services that can be traded in the national market at a competitive price $p_{k}^{*}$. These products can kick off complex competition and cooperation between the tradable sectors of different cities.

Suppose individuals have a $\log$ linear utility function over the two products: tradable products $\left(c_{k}\right)$, and nontradable products $\left(c_{u}\right)$ :

$$
u=\lambda_{k} \ln c_{k}+\lambda_{u} \ln c_{u},
$$

where $\lambda_{k}$ and $\lambda_{u}$ are the income elasticities for products from different sectors, here $\lambda_{k} \in(0,1) ; \lambda_{u} \in(0,1) ; \lambda_{k}+\lambda_{u}=1$. The utility function is homothetic. Unlike other industrial structure change models, our model about the adjustment of the two sectors in cities can obtain nonhomothetic results, without needing to assume that the demand is nonhomothetic. Production of the two sectors in cities can be, respectively, described as

$$
\begin{aligned}
& Y_{k}=A_{k} L_{k}^{1-\alpha}, \\
& Y_{u}=A_{u} L_{u}^{1-\alpha} .
\end{aligned}
$$

The functional form shows the existence of diminishing marginal products of labor in each production activity. $A_{k}$ 
and $A_{u}$ are the productivities of tradable and nontradable sectors, respectively; $L_{k}$ and $L_{u}$ are the proportions of the labor of tradable and nontradable sectors to the total labor, respectively $\left(L_{k}+L_{u}=1\right)$; the value of $\alpha$ is identical across all sectors.

The price $p_{k}^{*}$ of tradable products is determined by the competitive market in interregional trade, while the price $P_{u}$ of nontradable products is determined endogenously by the local market. If $q$ is income for an individual, the budget constraint for the individual is

$$
p_{k}^{*} c_{k}+p_{u} c_{u}=q .
$$

Considering the special background of urbanization and regional development in China, our theoretical model also takes account of the economic rent of CRN; so, the total income (flow) in the city from the economic rent of CRN is denoted by $R$, which is taken as given by individuals and producers. The economic rent of CRN is not fully regulated by the market. For example, the underdeveloped cities enjoy the extra nonmarket outputs, which comes from support policies like fiscal transfer, industrial subsidies, and preferential policy on infrastructure construction.

Economic rent of CRN changes the proportion of nontradable and tradable structures, and finally affects urban flow and outputs. For simplicity, we assume that the amount $R$ is distributed equally across all individuals. Although this assumption is not the case, inequality in the distribution does not change the basic results, as shown in the Gollin's [3] Web Theory.

Given the log-linear utility, the optimal choice for a consumer is for the expenditure share of income on nontradable products to equal its weight $\lambda_{u}$ in the utility function. The ideas of deduction are consistent with Gollin et al. [3]. Given that urban nontradables are only produced in the local market, total expenditure on them must equal the sum of production and the income from $R$. Let $\theta(0<\theta<1)$ be the proportion of CRN economic rent in nontradable sectors, so

$$
\lambda_{u} q=\theta R+p_{u} Y_{u}
$$

The larger $\theta$ is, the greater the role of government power in nontradable sectors is. The tradable products can be produced for the economy as a whole, traded from the rest of the nation. In addition, the city has the income partly from $R$, assuming balanced trade yields the following condition

$$
\lambda_{k} q=(1-\theta) R+p_{k}^{*} Y_{k}
$$

Since labor can flow freely between sectors, the wage rates of the sectors are equal in the equilibrium state. Then, the partial derivatives of formulas (2) and (3) satisfy

$$
(1-\alpha) p_{k}^{*} A_{k} L_{k}^{-\alpha}=(1-\alpha) p_{u} A_{u} L_{u}^{-\alpha} .
$$

From formulas (5)-(7), it can be derived that

$$
\begin{aligned}
L_{u}^{1-\alpha} & =\frac{\left[(1-\theta) \lambda_{u}-\theta \lambda_{k}\right] \cdot R}{P_{u} A_{u}\left[1-\lambda_{u}\left(P_{k}^{*} A_{k} / P_{u} A_{u}\right)^{1 / \alpha}\right]}, \\
L_{k}^{1-\alpha} & =\frac{\left[(1-\theta) \lambda_{u}-\theta \lambda_{k}\right] \cdot R}{P_{k}^{*} A_{k}\left[\left(P_{u} A_{u} / P_{k}^{*} A_{k}\right)^{1 / \alpha}-\lambda_{u}\right]} .
\end{aligned}
$$

Let $\rho=(1-\theta) \lambda_{u}-\theta \lambda_{k}$ be a constant combination between the consumption of the products from different sectors; $\tau=\left(P_{k}^{*} A_{k} / P_{u} A_{u}\right)$ be the productivity-price product (PPP) ratio of the two sectors, that is, the PPP of the tradable sector divided by that of the nontradable sector. Then, formulae (8) and (9) can be rewritten as

$$
\begin{aligned}
& L_{u}=\left[\frac{\rho R}{P_{u} A_{u}\left(1-\lambda_{u} \tau^{1 / \alpha}\right)}\right]^{(1 / 1-\alpha)}, \\
& L_{k}=\left\{\frac{\rho R}{P_{k}^{*} A_{k}\left[(1 / \tau)^{1 / \alpha}-\lambda_{u}\right]}\right\}^{(1 / 1-\alpha)} .
\end{aligned}
$$

From formulas (10) and (11), we have:

$$
\begin{gathered}
\text { (i) } \frac{\partial L_{u}}{\partial R}>0, \\
\frac{\partial L_{u}}{\partial \tau}>0, \\
\frac{\partial L_{u}}{\partial\left(P_{u} A_{u}\right)}>0, \\
\text { (ii) if }(1 / \tau)^{1 / \alpha}-\lambda_{u}>0, \text { then } \frac{\partial L_{k}}{\partial \tau}>0, \frac{\partial L_{k}}{\partial\left(P_{k}^{*} A_{k}\right)}<0, \\
\frac{\partial L_{k}}{\partial R}>0 .
\end{gathered}
$$
(13)

The following can be derived from formulae (12) and

(i) The adjustment of employment structure in cities depends on CRN economic rent $(R), \operatorname{PPP}\left(P_{k}^{*} A_{k}\right.$ or $\left.P_{u} A_{u}\right)$, and the PPP ratio of the two sectors $(\tau)$; the share of the nontradable sector in the employment structure $\left(L_{u}\right)$ increases with CRN economic rent $(R)$.

(ii) If underdeveloped regions choose to expand urban employment scale solely by increasing the productivity $A_{u}$ of the nontradable sector, the longterm effect will be very limited. The reason is that undeveloped cities have a high share of the nontradable sector; the rise of productivity $A_{u}$ of this sector will drag down.

(iii) $R, \tau$ and $P_{k}^{*} A_{k}$ have uncertain impacts on the share of the tradable sector in the employment structure $\left(L_{k}\right)$. The specific impacts depend on the original endowments of the cities. If $(1 / \tau)^{1 / \alpha}-\lambda_{u}>0$ or $(1 / \tau)^{1 / \alpha}-\lambda_{u}<0$, the variations in $R, \tau$, and $P_{k}^{*} A_{k}$ 
have different impacts on the adjustment of $L_{u}$. This further indicates that the change of the tradable sector involves complicated and intertwined issues like agglomeration economy, regional trade development, and the economic rent of CRN.

\section{CRN Economic Rent and Adjustment of Urban Production Structure}

3.1. Construction of Variables and Indicators. The statistical samples are selected from 277 cities in China. The original data from 2003 to 2018 are from China Urban Statistical Yearbook, Wind database, and China Stock Market \& Accounting Research Database. According to the ruling period of the two central governments, the statistical process is divided into two stages, 2004-2011 and 2012-2018. The Economic rent of CRN reflects the government intervention. Government intervention is measured by the ratio of fiscal expenditure to fiscal revenue (FEFR) in this paper. If there is a bigger FEFR in the city from 2004 to 2011, it means that the greater the government intervention, the greater the economic rent of CRN. The other covariates (urban features) are geographical location, income endowment, financial status, urban size, and agglomeration, and the specific construction process is shown in Table 1.

Considering the availability of data and following the principle of statistical yearbooks, the tradable sector was divided into the following parts: mining; manufacturing; information transmission, computer services, and software industry; finance and insurance industry; geological investigation, water conservation, and management industry; transportation, warehousing, post and telecommunications industry; scientific research and integrated technology services; cultural, sports, and entertainment industry; and neighborhood service and other service industry. The nontradable sector was divided into the following parts: wholesale and retail trade; accommodation and catering; real estate; rental and commercial services; educational and cultural broadcasting; and social services and public management.

All variables of 277 cities are preliminarily counted, and the detailed results are shown in Table 2.

3.2. Ridge Regression. In order to overcome the endogeneity of the traditional regression model, ridge regression, a classical machine learning technology, is used to analyze the relationship between covariates and dependent variables. As a data-driven algorithm, the basic idea of ridge regression is to reduce the regression coefficient to zero, so as to minimize the sum of RSS of regression model and penalty, so as to select a better set of covariates. The estimated values of ridge regression coefficients are obtained by minimizing the following equation:

$$
R=\sum_{i=1}^{n}\left[\left(L_{i}-C_{0}-\sum_{j=0}^{13}\left(\beta_{j} X_{i j}\right)\right]^{2}+\lambda \sum_{j=0}^{13} \beta_{j}^{2},\right.
$$

$L_{i}$ is the employment ratio of the two sectors in the city $i$, $X_{i j}$ is one of the features (shown in Table 2) in the city $i$, and $\lambda$ is the tuning parameter. Minimizing formula (14) to get the estimated coefficient of ridge regression, the 10fold Cross Validation is used to obtain the $\lambda$. After the variables are standardized, the change of MSE is shown in Figure 1. The figure on the left (MSE-A) describes the process of the ridge regression model in analyzing the data from 2004 to 2011, and the figure on the right (MSE-B) describes the data processing from 2012 to 2018. The minimum value of prediction model error is selected for Cross Validation, and estimation is shown in Table 3 .

Best $\lambda$ and MSE are small, and the empirical model of Ridge regression is effective. From the estimation of the whole sample in the whole period, the coefficients of FEFR (X9) are all negative, indicating that there is a linear correlation between the increase of government intervention and the declining share of tradable sector (nontradable sector increase), which indicates that China's economic rent of CRN has indeed affected the regional production structure. In fact, in the past 10 years, regional policy has become the main guiding ideology of China's high-level decisionmaking.

There is a nonlinear relationship between the productivity growth and production structure. In the initial stage, the coefficient of urban productivity growth $(X 13)$ is negative $(-0.383,-0.403$, and -0.307$)$ and becomes positive later, which means that during 2004-2011, the rapid increase of urban productivity and the increasing share of nontradable sectors appeared simultaneously, but this shortterm effect did not appear again in 2012-2018. This indirectly shows that the economic rent generated by China's regional policy can promote the growth of less-developed regions and employment in nontradable sectors in the short term, but in the long run, the productivity growth of nontradable sectors is relatively slower, and the growth of developed regions with higher proportion of tradable sectors will be higher, which leads to the complex convergence in different regions.

On the interaction term, FEFR $\times$ Income endowment $(X 9 \times X 3)$ and FEFR $\times$ Productivity growth $(X 9 \times X 13)$ are negative. Specifically, the impact of one unit of productivity improvement on production structure (tradables/nontradables) is $-0.017 \times \mathrm{X} 9$ and $-0.030 \times \mathrm{X} 9$. Therefore, the more the government intervention, the higher the nontradable share.

\section{Robust Analysis from CRN Gravity Flows}

In order to make the results in the previous section more robust, we use the method of combining urban gravity flows and social network analysis (SNA) to carry out further analysis. This statistical technology of SNA can effectively solve the endogeneity of linear model, especially the endogeneity of mutual causality. Mainly, we construct a new Gravity model to obtain direction data. 
TABLE 1: Description of indicators.

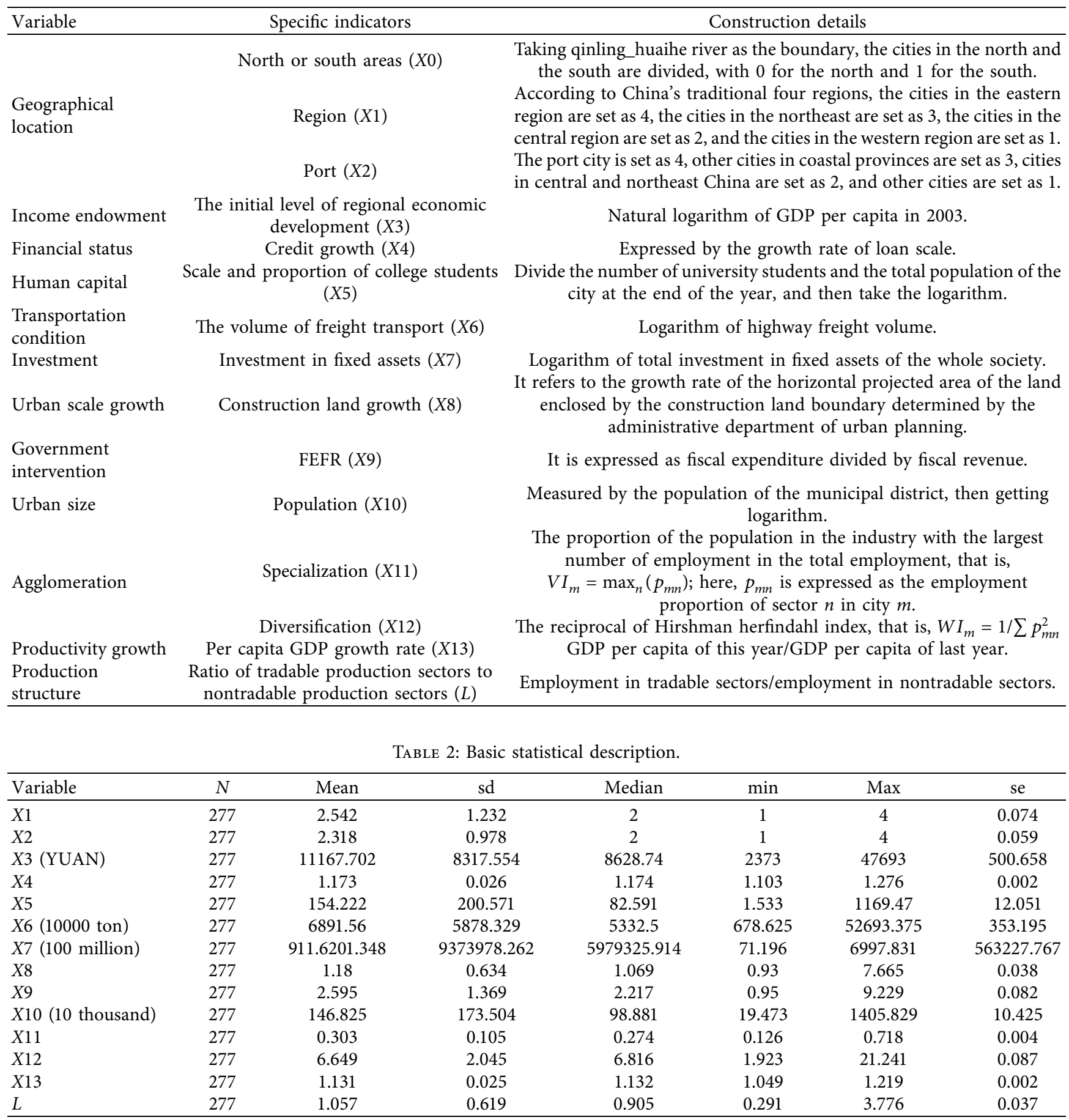

Note: these data are raw data without logarithmic processing.

4.1. Statistics on Gravity Flows. In CRN, complex flow links have been formed by the complicated trade network, traffic network, and people-to-people interactions between cities. The flows in CRN are a composite form of people flow, logistics, and capital flow. Therefore, it is reasonable to adopt the gravity model to analyze the evolution trends of CRN's flow changes and spatial relational structure.
Referring to the handling of the gravity model by Kolaczy and Csárdi [21], and Liu et al. [22], this paper derives from the above basic theories that the CRN flows are affected by $\operatorname{PPP} \pi$, city scale $Q$, city distance $d$, and employment ratio $l$ of the two sectors.

Then, the gravity flow from city $j$ to city $i$ in CRN can be expressed as 


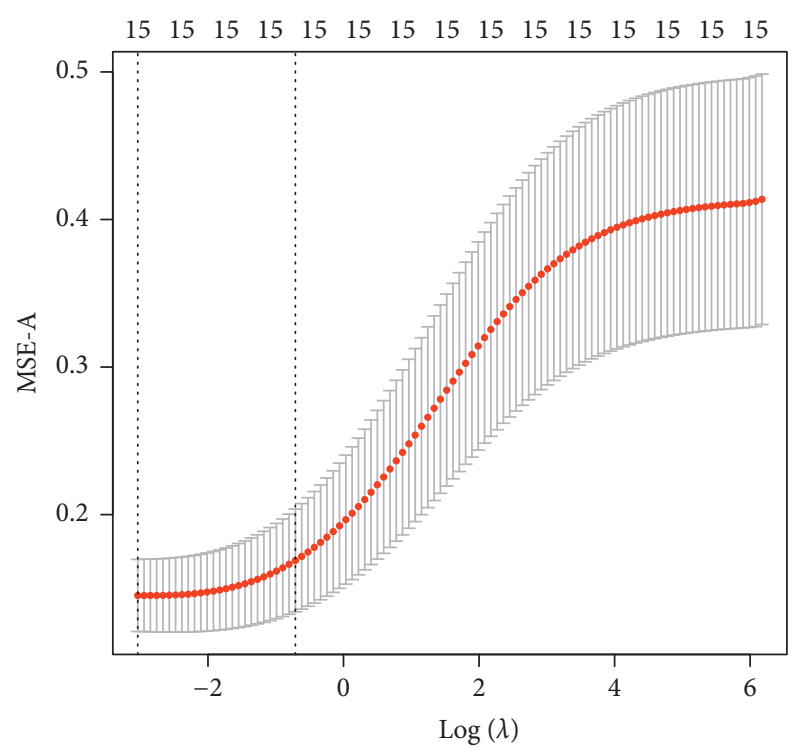

(a)

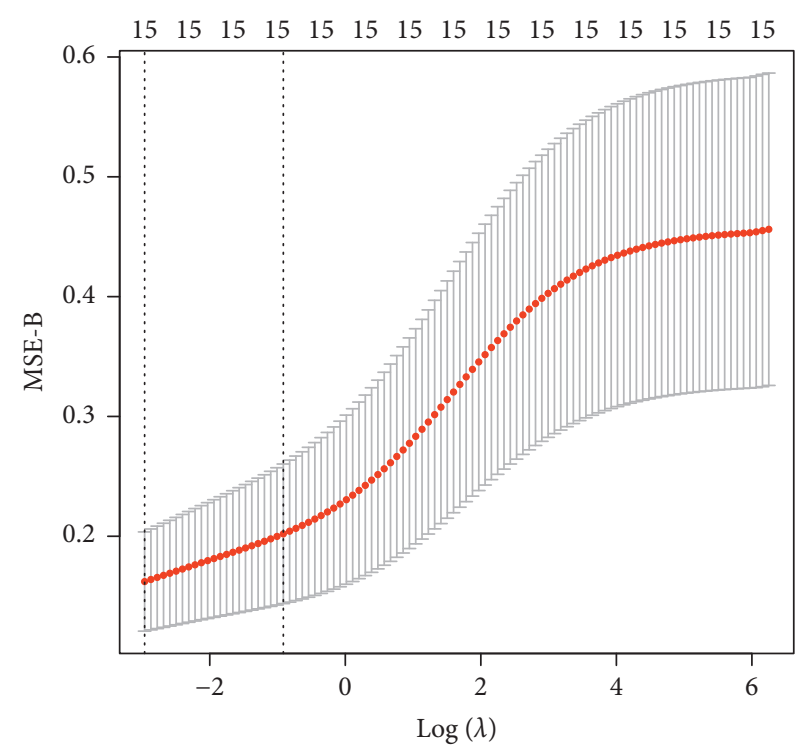

(b)

Figure 1: Relationship between tuning parameter and test error.

TABLE 3: Ridge regression result.

\begin{tabular}{lcccccc}
\hline & \multicolumn{3}{c}{$2012 \sim 2018$} & \multicolumn{3}{c}{$2004 \sim 2011$} \\
\hline (Intercept) & -1.336 & -1.243 & -1.330 & -1.501 & -1.383 & -1.521 \\
$X 0$ & -0.098 & -0.099 & -0.098 & -0.192 & -0.201 & -0.199 \\
$X 1$ & 0.006 & 0.006 & 0.006 & -0.001 & -0.002 & -0.001 \\
$X 2$ & -0.014 & -0.015 & -0.015 & -0.041 & -0.045 & -0.044 \\
$X 3$ & 0.259 & 0.261 & 0.257 & 0.298 & 0.308 & 0.302 \\
$X 4$ & -1.670 & -1.685 & -1.672 & 1.436 & 1.474 & 1.492 \\
$X 5$ & 0.029 & 0.028 & 0.029 & 0.060 & 0.060 & 0.060 \\
$X 6$ & 0.081 & 0.078 & 0.080 & 0.021 & 0.020 & 0.022 \\
$X 7$ & -0.067 & -0.069 & -0.068 & -0.120 & -0.133 & -0.133 \\
$X 8$ & 0.012 & 0.013 & 0.013 & 0.010 & 0.011 & 0.011 \\
$X 9$ & -0.042 & -0.010 & -0.027 & -0.078 & -0.035 & -0.049 \\
$X 10$ & 0.021 & 0.022 & 0.021 & 0.072 & 0.078 & 0.079 \\
$X 11$ & 3.746 & 3.742 & 3.743 & 1.776 & 1.799 & 1.802 \\
$X 12$ & 0.016 & 0.016 & 0.016 & -0.082 & -0.082 & -0.083 \\
$X 13$ & 0.850 & 0.846 & 0.893 & -0.383 & -0.403 & -0.307 \\
$X 3 \times X 9$ & & -0.005 & & & -0.006 & \\
$X 9 \times X 13$ & & & -0.017 & & & -0.030 \\
Best lamda & 0.052 & 0.052 & 0.052 & 0.063 & 0.048 & 0.048 \\
MSE & 0.259 & 0.259 & 0.259 & 0.118 & 0.116 & 0.117 \\
\hline
\end{tabular}

Note: All variables are taken as the average of the corresponding stage (2012-2018, or 2004-2011). Dependent variable is L. The Variables were standardized.

$$
\begin{aligned}
f_{i j} & =\sigma_{i j} \frac{\left(l_{i} Q_{i} \pi_{i}\right)^{1 / 2} \cdot\left(l_{j} Q_{j} \pi_{j}\right)^{1 / 2}}{d_{i j}^{2}} \\
\sigma_{i j} & =\frac{\pi_{i}}{\pi_{i}+\pi_{j}},
\end{aligned}
$$

where $Q_{i}$ and $Q_{j}$ are the scales of cities $i$ and $j$, respectively, which are represented by population; $\pi_{i}$ and $\pi_{j}$ are the PPPs of cities $i$ and $j$, respectively, which are represented by percapita GDP; $d_{i j}$ is the geographical distance between cities $i$ and $j$, which is measured by the actual expressway distance between them on Baidu Map; $\sigma_{i j}$ and $\sigma_{j i}$ are CRN gravity coefficients, which reflect the contributions of cities $i$ and $j$ to the gravity flow $f_{i j}$ between them, respectively. Through the use of gravity coefficient, gravity flows of CRN has directionality, which is defined the relational data in this paper. The advantage of relational data is more realistic because the flows of Beijing to Zhengzhou are obviously different from that of Zhengzhou to Beijing. In addition, social network analysis (SNA) is carried out through relational data, and the endogenous problem of regression is not considered.

Considering the availability of CRN data and the complexity of computation, 39 representative cities on different levels were selected from different regions to discuss the CRN of China: Beijing, Xingtai, Changzhi, Chifeng, Fuxin, Siping, Qiqihar, Shanghai, Yancheng, Suqian, Hangzhou, Lishui, Huainan, Putian, Pingxiang, Heze, Zhengzhou, Kaifeng, Wuhan, Huangshi, Changsha, Hengyang, Yongzhou, Guangzhou, Shaoguan, Shenzhen, Shantou, Nanning, Qinzhou, Chengdu, Zhangzhou, Zunyi, Kunming, Zhaotong, Xi'an, Weinan, Lanzhou, Tianshui, and Xining.

To capture the latest spatial correlations and sector restructuring laws of Chinese cities, this paper sorts out and models the CRN gravity flows of the 39 cities in 2003, 2011, and 2018, using 1482 gravity flow data each year. These 39 cities evenly divide Chinese cities into three development levels and even geographical distribution. The years 2003 and 2011 are the turning time for the adjustment of the central governments, respectively. Based on the data availability of existing variables, the latest is 2018 . The basic data used to measure the CRN gravity flows were collected from China Statistical Yearbooks and China City Statistical Yearbooks, and processed by social network analysis (SNA) method. Table 4 presents the variables and their meanings 
TABLE 4: Relevant variables.

\begin{tabular}{lcc}
\hline Name & Abbreviation & Meaning \\
\hline Employment structure of city $i$ & lnemployment_i & Employment ratio between the two sectors in city $i$ \\
Employment structure of city $j$ & lnemployment_j & Employment ratio between the two sectors in city $j$ \\
Scale of city $i$ & lncitysize_ $i$ & Population of the districts administered by city $i$ \\
Scale of city $j$ & lncitysize_j $j$ & Population of the districts administered by $j$ \\
Employment structure ratio between the two cities & lnemployment_ij & Employment structure of city $i$ divided by that of city $j$ \\
Distance between the two cities & lndistance_ij & Expressway distance between cities $i$ and $j$ on Baidu Map \\
Per-capita GDP ratio between the two cities & $\operatorname{lngdp\_ ij}$ & Per-capita GDP of city $i$ divided by that of city $j$ \\
Gravity flow from city $j$ to city $i$ & $\ln f_{i j}$ & $f_{i j}=\sigma_{i j}\left(\left(l_{i} Q_{i} \pi_{i}\right)^{1 / 2} \cdot\left(l_{j} Q_{j} \pi_{j}\right)^{1 / 2} / d_{i j}^{2}\right)$ \\
\hline
\end{tabular}

involved in the empirical analysis. For the lack of space, the statistical results of these variables are not fully displayed. Any interested reader can contact the authors for the full results or the source code.

4.2. Gravity Flows and CRN. The processing of gravity flows is the core work of CRN analysis. The modeling and prediction of CRN topology were carried out mainly based on the ideas of Kolaczy and Csárdi [21] on SNA. The difference lies in gravity coefficient $\sigma$; a pseudo count was added to the flow data, making the latter more in line with independent Poisson's distribution; further, the Newton-Raphson algorithm was optimized to a certain extent.

Here, a CRN is represented by $G=(V, E) . G$ is a directed graph, because each gravity flow is directed from the start point to the end point. The edges in $G$ are also referred to as links. This research focuses on the start-end point matrix $F=$ $\left[f_{i j}\right]$ composed of the gravity flows from node $i$ to node $j$. This matrix is also known as gravity flow matrix.

In CRN, each directed edge was assigned a weight, which was calculated by multiplying each $f_{i j}$ with 5 and then divided by the minimum value of $F=\left[f_{i j}\right]$. Since 39 city nodes on different levels were selected, there were $39 \times 38=1,482$ CRN gravity flows each year. The relations between the city nodes in 2003, 2011, and 2018 can be visualized as Figures 2-4, respectively. Note that the size of each circle reflects the total gravity flow of the corresponding city; the white and light blue parts of the circle represent the gravity inflow and gravity outflow, respectively; the link width stands for the flow from the start point to the end point.

Figures 2-4 show that, as time goes on, the inflow of gravity flow to cities in the south is generally better than that in the north, especially in cities with strong innovation ability and prosperous tradable sectors such as Shenzhen, Guangzhou, and Hangzhou, while the inflow to cities in the north is relatively weak, although some national central cities (such as Zhengzhou) performed well.

Judging by the size of the circles in CRN, central cities like Shenzhen, Guangzhou, Shanghai, Hangzhou, Xi'an, Zhengzhou, and Changsha had very large gravity flows, while the vast majority of central and western cities and other prefectural-level cities had very small gravity flows; the latter cities also faced a high share of outflow.

Among the cities with large gravity flows, Shenzhen had always maintained a very large flow. Hangzhou saw a gradual increase in the inflow-outflow ratio. The city achieves eye-catching gravity flow and economic vibrance as a result of its digital economic boom in the recent decade. The inflow-outflow ratio of Guangzhou decreased to a certain extent, which has much to do with the transformation and upgrading of traditional industries, and the replacement of low-end industries with high-end ones. Despite that, Guangzhou still boasted a large gravity flow. As a national central city, Zhengzhou had a relatively large gravity flow, but its inflow-outflow ratio first increased and then decreased. In recent years, the inflow-outflow ratio of Shanghai dropped a little bit. As for Beijing, the gravity flow was not as large as expected; an important reason is the high proportion of nontradable sector in the employment structure, especially the headquarters of central enterprises or public management departments of government organs; of course, there are some ambiguities about the statistical data of the parts of nontradable sectors.

\section{Testing and Analysis of CRN Gravity Flow}

The gravity flows obtained by formula (15) agree well with the city development in China. However, the gravity model $f_{i j}$ needs more supports from theoretical and empirical tests. After all, it is not as naturally acceptable as Newton's law of Gravitation. Therefore, the MLE of independent Poisson's distribution was adopted to further test and correct the model.

To further quantify and correct the gravity flow model, this paper selects the MLE to derive that model. Similar to logistic regression, the MLE uses the IWLS method derived from the Newton-Raphson method. After the count form had been set, the CRN flow $f_{i j}$ was assumed to obey independent Poisson's distribution. The mean function of $f_{i j}$ can be defined as

$$
\mu_{i j}=E\left(f_{i j}\right)=m_{D}\left(\phi_{i j}\right) m_{S}\left(c_{i j}\right) .
$$

Then, the benchmark log linear model with Poisson's distribution can be expressed as

$$
\ln f_{i j}=\alpha_{0}+\alpha_{1} \ln m_{D}\left(\phi_{i j}\right)+\alpha_{2} \ln m_{S}\left(c_{i j}\right),
$$

where $m_{D}\left(\varphi_{i j}\right)$ is the relative value of an economic variable between cities $i$ and $j$ (e.g., the per-capita GDP ratio $m_{1}\left(\varphi_{i j}\right)$ and the employment structure ratio $m_{2}\left(\varphi_{i j}\right) ; c_{i j}$ is a vector representing the separation attribute of the relationship between the two cities, e.g., distance or cost. To further highlight the CRN correlations and economic rent issue, 


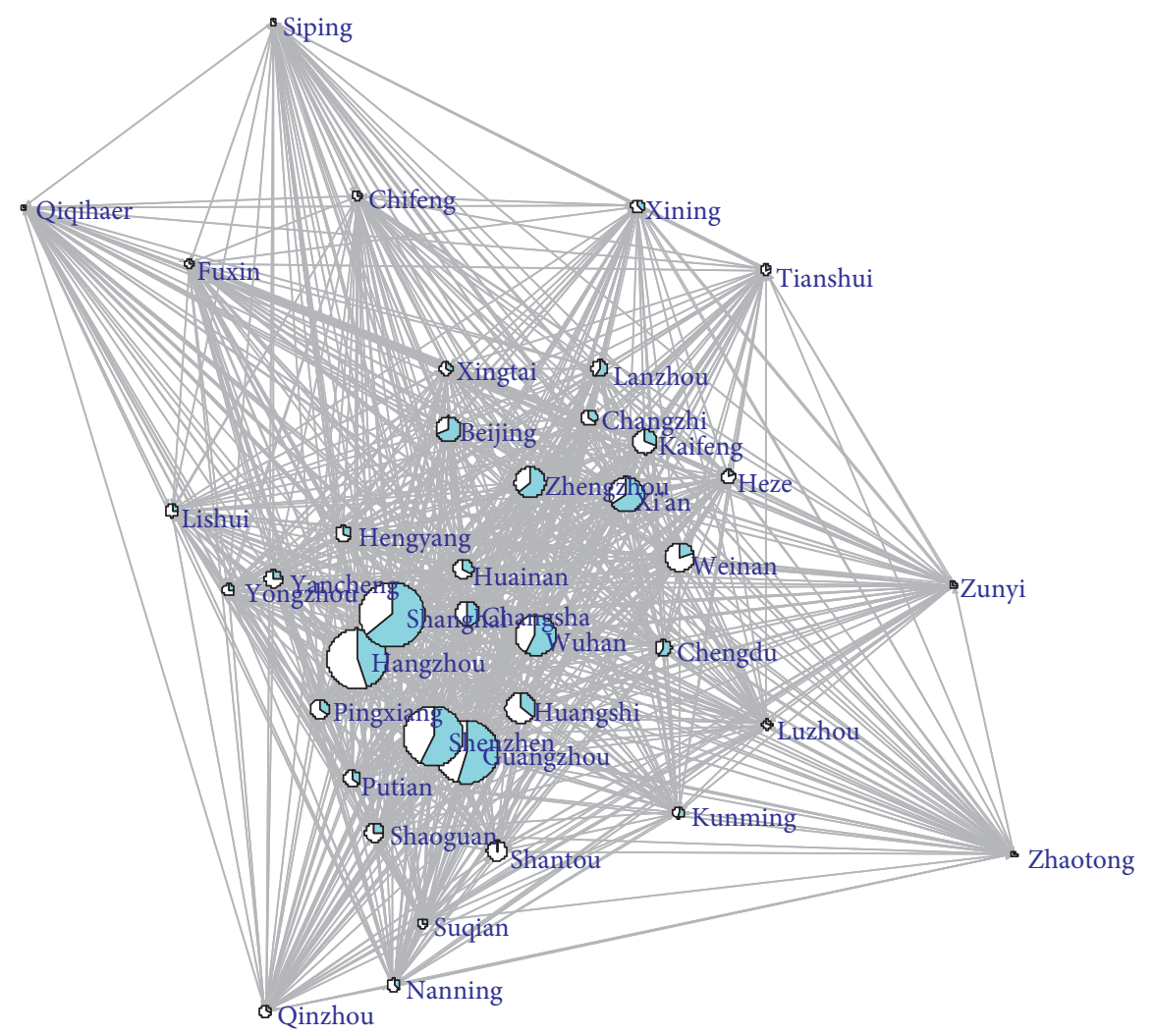

FIgURE 2: Visualized CRN gravity flows in 2003.

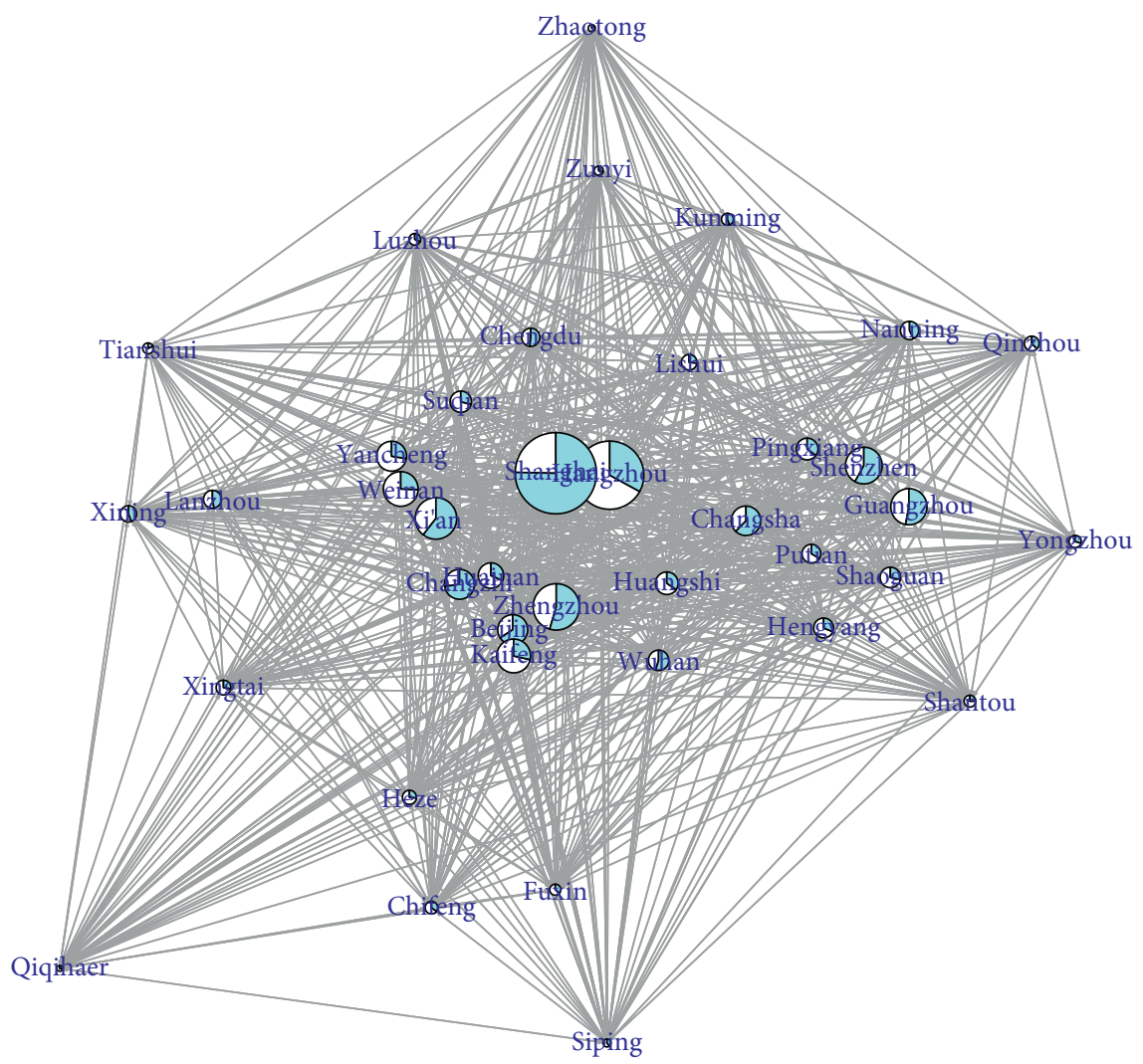

FIgURE 3: Visualized CRN gravity flows in 2011. 


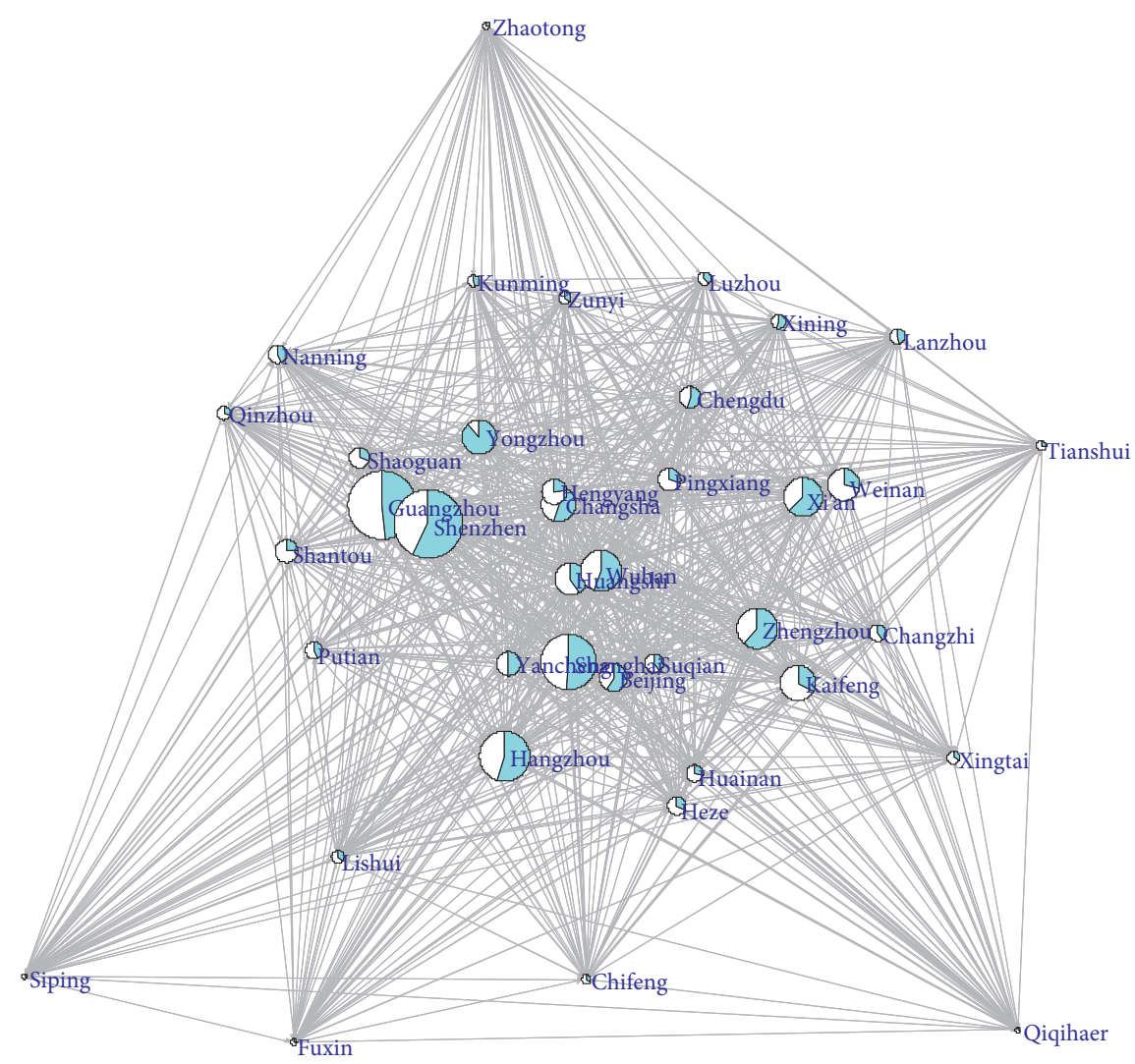

Figure 4: Visualized CRN gravity flows in 2018.

generalization is necessary to test the GRN gravity flow model, including the assumption of the independence between start point effect and end point effect. The generalized linear model can be expressed as

$$
\begin{gathered}
\ln \mu_{i j}=\ln h(i)+\ln h(j)+\beta_{1} \ln m_{1}\left(\phi_{i j}\right)+\beta_{2} \ln m_{2}\left(\phi_{i j}\right)+\beta_{3} \ln m_{S}\left(c_{i j}\right) \\
\eta(\mu)=\sum_{i, j \in \ell \times \ell} f_{i j} \ln \mu_{i j}-\mu_{i j} .
\end{gathered}
$$

of cities $i$ and $j$, respectively. During the empirical test, these attributes were parametrized, such as taking the logarithms. Since $f_{i j}$ is an independent variable with Poisson distribution and the mean of $\mu_{i j}=E\left(f_{i j}\right)$, the most reasonable derivation approach is the MLE. Following the ideas of Poisson's distribution and MLE, the log-likelihood function in Poisson's distribution related to $\mu$ can be established as
Substituting formula (19) into formula (18), the MLE values of $h(i), h(j), \beta_{1}, \beta_{2}$, and $\beta_{3}$ can be obtained by making their partial derivatives zero. Then,

$$
\widehat{\mu}_{i j}=\ln \widehat{h}(i) \cdot \ln \widehat{h}(j) \exp \left[\widehat{\beta}_{1} \ln m_{1}\left(\phi_{i j}\right)+\widehat{\beta}_{2} \ln m_{2}\left(\phi_{i j}\right)+\widehat{\beta}_{3} \ln m_{S}\left(c_{i j}\right)\right]
$$

According to the conclusions of Kolaczy and Csárdi [21], the unique solutions of $\widehat{\mu}_{i j}, \widehat{\beta}_{1}, \widehat{\beta}_{2}$ and $\widehat{\beta}_{3}$ can be estimated well under nonextreme conditions. Although $\ln \widehat{h}(i)$ and $\ln \widehat{h}(j)$ could be overparametrized and generate redundant degrees of freedom (DOFs), the core conclusions will not be affected, because the MLE approach of our model uses the IWLS method derived from the Newton-Raphson method (similar to logistics regression). In addition, internalities and multicollinearity were largely overcome by adopting the data on directed relations in our log linear model, as well as the IWLS method derived from the Newton-Raphson method in the glm () function of $R$ language. Furthermore, the mean of per-capita GDP ratio between the two cities $\kappa_{i j}=E\left(g d p \_i j\right)$ was estimated under similar principles. The fitted results are listed in Tables 5 and 6. 
TABLE 5: Results of generalized linear model for mean CRN flow.

\begin{tabular}{lccc}
\hline Explanatory variables & $(2003)$ & $\ln \mu_{i j}$ & $(2011)$ \\
\hline$C$ & $2.585^{* * *}(17.866)$ & $2.529^{* * *}(16.638)$ & $(2018)$ \\
lnemployment_ $i$ & $0.061^{* * *}(3.534)$ & $0.093^{* * *}(3.567)$ & $0.506^{* * *}(16.191)$ \\
lnemployment_j & $0.051^{* *}(2.948)$ & $0.068^{* *}(2.576)$ & $0.094^{* *}(3.035)$ \\
lncitysize_ $i$ & $0.144^{* * *}(11.007)$ & $0.134^{* * *}(10.033)$ & $0.075^{*}(2.439)$ \\
lncitysize_j $j$ & $0.092^{* * *}(7.544)$ & $0.101^{* * *}(7.764)$ & $0.151^{* * *}(10.555)$ \\
lngdp_ij $i j$ & $0.019^{* * *}(3.909)$ & $0.030^{* *}(3.068)$ & $0.086^{* * *}(6.321)$ \\
lndistance_ij & $-0.161^{* * *}(-20.552)$ & $-0.164^{* * *}(-20.847)$ & $0.028^{* *}(2.753)$ \\
DOF & & 1,481 & $-0.163^{* * *}(-20.640)$ \\
\hline
\end{tabular}

TABLE 6: Results of generalized linear model for per-capita GDP ratio.

\begin{tabular}{lccc}
\hline Explanatory variables & \multicolumn{1}{c}{$\ln \kappa_{i j}$} & $(2018)$ \\
\hline$C$ & $(2003)$ & $(2011)$ & $1.009^{* * *}(4.509)$ \\
lncitysize_ $i$ & $1.272^{* * *}(5.985)$ & $0.097^{* * *}(6.933)$ & $0.116^{* * * *}(5.225)$ \\
lncitysize_j $j$ & $0.117^{* * *}(9.160)$ & $-0.101^{* * *}(-6.928)$ & $-0.127^{* * *}(-8.596)$ \\
lnemployment_ij & $-0.126^{* * *}(-9.168)$ & $0.098^{* *}(3.148)$ & $0.068^{*}(2.435)$ \\
lndistance_ij & $0.062^{*}(2.274)$ & $0.001(0.111)$ & $0.0004(0.042)$ \\
DOF & $0.001(0.151)$ & 1,481 & \\
\hline
\end{tabular}

Note: In Tables 5 and 6, “***," “**, " “*," and “.” represent the significance at the levels of $0.1 \%, 1 \%, 5 \%$, and $10 \%$, respectively; the bracketed numbers are $Z$ scores; Columns (2003), (2011), and (2018) report the generalized linear estimation results on each Poisson's distribution for 2003, 2011, and 2018, respectively.

The empirical results in Table 5 show that, among the estimations of the generalized linear model for mean CRN flow $\mu_{i j}$ each year, the coefficients of explained variables like city scale, distance, and employment structure were very significant. When the employment ratio of the two sectors in city $i$, i.e., the employment structure ln employment_ $i$ of city $i$, increased, the mean gravity flow from city $j$ to city $i$ would rise; When that ratio in city $j$ increased, the mean gravity flow from city $j$ to city $i$ would rise, too, but by a much smaller amplitude. In 2003, 2011, and 2018, the coefficients of ln employment $\_i$ were $0.061,0.093$, and 0.094, respectively, while the coefficients of ln employment $j$ were $0.051,0.068$, and 0.075 , respectively. However, the employment structures of the two cities had a common feature: with the elapse of time, the employment ratio of the two sectors contributed more and more to CRN gravity flow. This means developed cities with a high portion of tradable sector tend to witness a relatively fast growth of gravity flow. This trend became increasingly prominent in recent years.

According to the fitted results on mean per-capita GDP ratio in Table 6 , the coefficient of distance, one of the three explained variables, was not significant, but the coefficients of CRN gravity flow and employment structure ratio were significantly positive. In 2003, 2011, and 2018, the coefficients of employment structure ratio were $0.062,0.098$, and 0.068 , respectively. This directly supports the expectation that the productivity will grow relatively fast in cities with a large share of the tradable sector. For underdeveloped cities with a heavy presence of the nontradable sector, two results could be deduced: on the one hand, CRN economic rent could expand the scale of the nontradable sector, increasing its share in employment structure; since the products of this sector are not tradable, the overall productivity of the cities dominated by the nontradable sector will grow slowly than the cities with a large share of tradable sector; as a result, the regional development will converge at a slower speed. On the other hand, the rising productivity of the nontradable sector will reduce the prices in some parts in that sector; Thus, underdeveloped regions could not effectively expand urban employment scale solely by improving the productivity of the nontradable sector.

Next, we further test the reliability of the results and the performance of the model shown in Tables 5 and 6 . The left subgraph of Figure 5 shows the relationship between the fitting value $\widehat{\mu}_{i j}$ of the generalized linear model (e.g. formula (18)) and the statistical CRN gravity flows $f_{i j}$. Because the dynamic range of the involved values is very wide, the two groups of data are processed in the form of double logarithm coordinates. Prediction effect of the model is better when CRN gravity flow is large,

The right side of Figure 5 shows the comparison between the relative error and CRN gravity flows, also using the double logarithmic coordinates. It can be seen clearly in the figure that $\log \left[\left(f_{i j}-\widehat{\mu}_{i j}\right) / f_{i j}\right] \leq 0$ accounts for most of the proportion, that is, the relative error of model on CRN gravity flows is very small, and the relative error does not change greatly with the CRN gravity flows. This means that it 


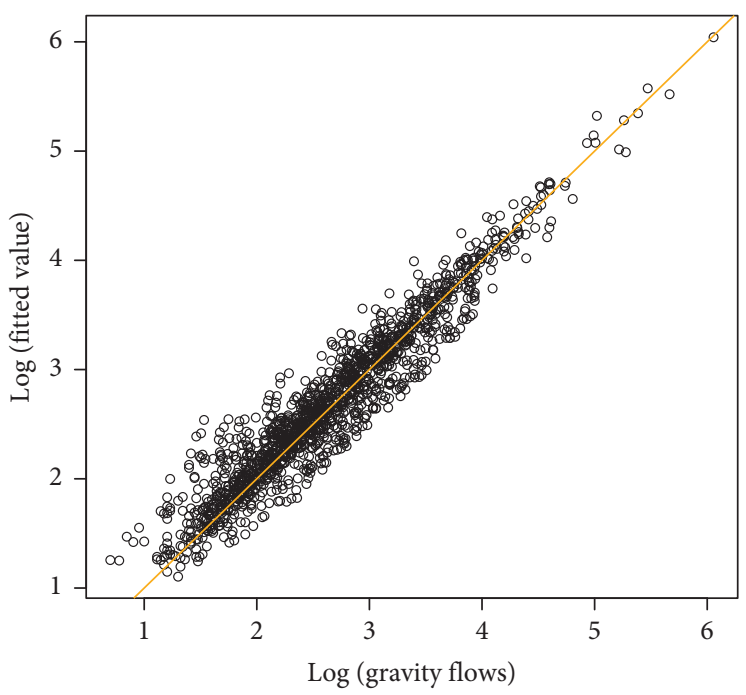

(a)

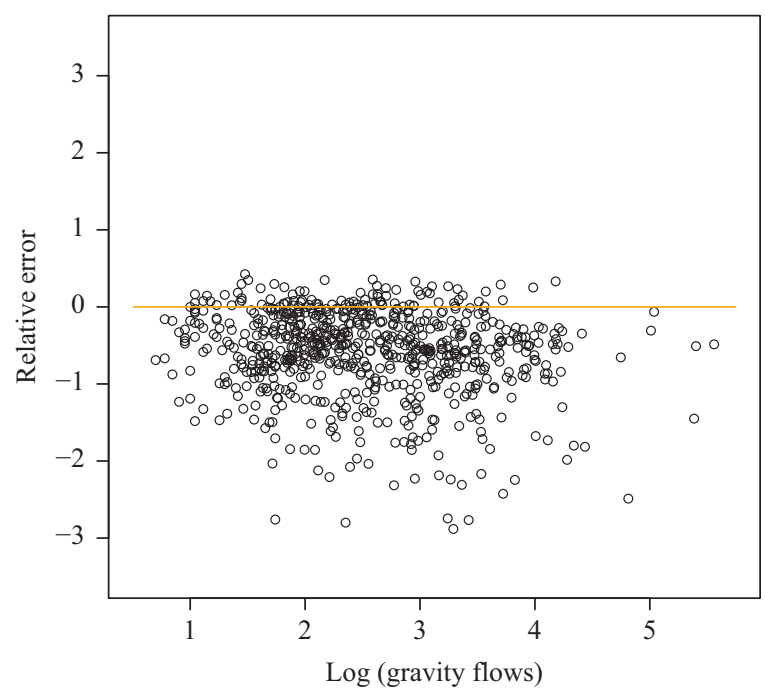

(b)

Figure 5: Model error on CRN gravity flows. Note: The abscissa uses natural logarithm, and the solid line reference system distribution of the left and right figures is $y=x$ and $y=0$, respectively.

is effective to analyze the structure adjustment and regional development of China's urban production sector by using the New Gravity model.

\section{Conclusions}

In China, the policies on new urbanization and balanced regional development have created a highly interactive network between people flow, logistics, and capital flow. Against this backdrop, a CRN gravity flow model was constructed through a social network analysis on the gravity flows between Chinese cities in different regions and on different levels, coupled with the data on other directed relations. The authors drew the following conclusions:

First, this paper establishes a statistical model of CRN gravity flows with the characteristics of urbanization in China, and proves the effectiveness of the model in analyzing the correlations and flow trend of CRN. Unlike traditional gravity models centering on population or per-capita GDP, our new gravity flow model includes novel variables like the ratio between the two sectors and the gravity coefficient. Besides, a generalized linear model was built on the data of directed relations for fitting. The estimated coefficients of the model were very significant, and the relative error was reasonable.

Second, the slowing convergence of regional development in China is directly driven by the relative adjustment of the production sector: the proportion of the tradable sector continues to increase in developed cities, while that of nontradable sector keeps growing in redeveloped regions. Compared with underdeveloped cities, developed cities have a high share of the tradable sector, a fast-growing CRN gravity inflow, and a rapid increase of productivity; these advantages became increasingly obvious in recent years. This paper offers sufficient theoretical and empirical statistics about the close correlation between the gravity inflow of a city and the employment ratio of that city, i.e., the ratio between the two sectors. Based on the relevant data, the coefficients of the employment ratio were $0.061,0.093$, and 0.094 in 2003, 2011, and 2018, respectively; the coefficient increased clearly over time. Therefore, underdeveloped regions could not effectively expand urban employment scale solely by improving the productivity of the nontradable sector.

In recent years, the accelerated urbanization process in China has brought about complex changes to the people flow, logistics, and capital flow in CRN. The spatial correlations bred by CRN economic rent have moved beyond the research scope of nearest neighbor distance in new economic geography or spatial econometrics. Therefore, our new CRN gravity flow model can effectively process directed flows and optimize the relevant algorithms, greatly promote the construction of equal and high-quality cities for all residents, and provide the theoretical foundation for coordinating regional development and pursuing collaborative development of future smart cities. Despite selecting 39 representative cities in a well-connected CRN, this paper does not consider a massive amount of data network maps (e.g., degree centrality, betweenness centrality, and closeness centrality). At present, this model can only consider the special CRN in China. Next, we will discuss the feasibility of this model using more data from more countries.

\section{Data Availability}

The data used to support the findings of this study are available from the corresponding author upon request.

\section{Conflicts of Interest}

The authors declare that they have no conflicts of interest. 


\section{Acknowledgments}

This research was supported by the Youth Project of National Social Science Fund of China (Grant No.: 18CJL032) under the program titled "Sustainability of Regional Balance Policy in the Great Transformation of Urbanization.

\section{References}

[1] P. A. Samuelson, "Theoretical notes on trade problems," The Review of Economics and Statistics, vol. 46, no. 2, pp. 145-154, 1964.

[2] B. Balassa, "The purchasing-power parity doctrine: a reappraisal," Journal of Political Economy, vol. 72, no. 6, pp. 584-596, 1964.

[3] D. Gollin, R. Jedwab, and D. Vollrath, "Urbanization with and without industrialization," Journal of Economic Growth, vol. 21, no. 1, pp. 35-70, 2016.

[4] Z. G. Hu, Y. Z. Wang, and G. J. Yin, "Selection, agglomeration and disparities of urban productivity," Economic Review, vol. 2, pp. 3-16, 2017.

[5] S. H. Wei, Y. Yang, and L. L. Chen, "City administrative hierarchy, differential growth of city size and evolution of urban system in China," China Industrial Economics, vol. 7, pp. 5-23, 2020.

[6] W. Q. Pan, “"Regional linkage and the spatial spillover effects on regional economic growth in China," Economic Research Journal, vol. 1, pp. 54-65, 2012.

[7] T. L. An and C. Yang, "How the internet is reshaping China's economic geography: micro mechanism and macro effects," Economic Research Journal, vol. 55, no. 2, pp. 4-19, 2020.

[8] B. Li, D. M. Guo, and S. Q. Liu, "City size, internal migration and non-tradable goods variety: evidence from MeituanDianping," Economic Research Journal, vol. 54, no. 1, pp. 150-164, 2020.

[9] R. Milo, S. Shen-Orr, S. Itzkovitz et al., "Network motifs: simple building blocks of complex networks," Science, vol. 298, no. 5594, pp. 824-827, 2002.

[10] Y. J. Kang and X. H. Liu, "Firm heterogeneity in the tradable sector, export product diversification and real exchange rate," The Journal of World Economy, vol. 42, no. 12, pp. 166-188, 2019.

[11] R. Dix-Carneiro and B. K. Kovak, "Margins of labor market adjustment to trade," Journal of International Economics, vol. 117, pp. 125-142, 2019.

[12] B. He and S. Zhou, "Trade liberalization and regional employment adjustment: evidence from China's prefecture-level cities," The Journal of World Economy, vol. 42, no. 6, pp. 119-142, 2019.

[13] C. D. Shao, K. W. Li, and D. N. Su, "National value chain and interregional business cycle synchronization: evidence from China," Economic Research Journal, vol. 53, no. 3, pp. 187201, 2018.

[14] B. Q. Tang, B. Qiu, and S. Q. Sun, "Regional imbalances in China's growth in services: differences between the actual strength and optimal strength of IPR," Economic Research Journal, vol. 53, no. 8, pp. 147-162, 2018.

[15] G. Fagiolo, J. Reyes, and S. Schiavo, “"World-trade web: topological properties, dynamics and evolution," Physical Review, vol. 79, no. 3, Article ID 036115, 2019.

[16] P. J. Taylor, M. Hoyler, and R. Verbruggen, "External urban relational process: introducing central flow theory to complement central place theory," Urban Studies, vol. 47, no. 13, pp. 2803-2818, 2010.
[17] G. Fagiolo, "The international-trade network: gravity equations and topological properties," Journal of Economic Interaction and Coordination, vol. 5, no. 1, pp. 1-25, 2010.

[18] J. Li, S. Chen, G. H. Wan, and C. M. Fu, "Study on the spatial correlation and explanation of regional economic growth in China-_based on analytic network process," Economic Research Journal,vol. 49, no. 11, pp. 4-16, 2014.

[19] S. Wasserman and K. Faust, Social Network Analysis: Methods and Applications, Cambridge University Press, , Cambridge, England, 1994.

[20] J. Scott, Social Network Analysis: A Handbook, Sage, California, CA, USA, 2017.

[21] E. D. Kolaczy and G. Csárdi, Statistical Analysis of Network Data with R, Springer, New York, NY, USA, 2014.

[22] H. J. Liu, C. M. Liu, and Y. N. Sun, "Spatial correlation network structure of energy consumption and its effect in China," China Industrial Economics, vol. 5, pp. 83-95, 2015. 